\title{
Robust BDCM Sensorless Control With Position-Dependent Load Torque
}

\author{
*Chih-Kai Huang, *Pei-Yu,Yu, **Hung-Chi Chen (IEEE Member), \\ *Industrial Technology Research Institute, HsinChu, Taiwan. \\ Jeff.Huang@itri.org.tw \\ **Department of Electrical and Control Engineering, \\ National Chiao Tung University, HsinChu, Taiwan.
}

\begin{abstract}
This paper presents the robust sensorless operation for brushless DC motors (BDCMs) loaded by the positiondependent load where the load torque is a function of the rotor angle (not electrical angle). Without any position sensor, the reliable position signals are generated from the motor terminal voltages through sensorless circuits. In order to reduce the speed variations especially in the low speed, an additional gain to adjust output voltage is included in the speed loop to compensate the repetitive position-dependent load. In first, the effects of positiondependent load on the speed variations and the commutation intervals are studied. It shows that we can estimate the positiondependent load from the commutation intervals. Then, we introduce the used sensorless scheme based on sensing terminal voltage. Finally, some simulated results have been given to demonstrate the robust performance of the proposed sensorless control for the position-dependent loads.
\end{abstract}

\section{INTRODUCTION}

During the last decades, more and more attentions are put on the energy-saving technology and the high-efficiency products such as the air conditioner and refrigerators. However, the development of the variable-speed compressors has been regarded as the key to increase the efficiency of the overall refrigeration system. Recently, there are two main trends in the development of variable-speed refrigeration systems: (i) the utilization of high-efficiency compressor motors and (ii) the application of low-production-cost compressors.

Compared to all the variable-speed motors like the induction motors (IMs), the synchronous reluctance motors (SRMs) and the brushless DC motors (BDCMs) [1], the latter two are free from the exciting loss and rotor copper loss and, therefore, possess higher efficiency than the widely used induction motors (IMs). Moreover, because of the unusual power circuits for SRMs, BDCMs are gradually utilized as the high-efficiency compressor motors. In order to operate BDCMS efficiently, the reliable rotor positions are required to commutate the winding current adequately. Unfortunately, in the refrigeration application, the high-temperature refrigerants will surround the compressor motor and the general rotor position sensor such as Hall elements cannot be used. In order to operate BDCMs without any position sensors, developing sensorless control is important.

To solve it, many sensorless control methods had been proposed to replace the function of the position sensor. They can be divided into two groups according to the waveforms of the drawn motor currents: one group is discontinuous winding currents (or called square-wave currents) and the other is continuous currents (or called sinusoidal-wave currents). Since there always is a floating motor terminal in the former group, some schemes including sensing terminal voltages and diode currents are used to directly obtain the rotor positions [2-4].

In addition, there are several types of compressors in the market for various refrigeration systems and cooling capacities. However, from the motor's point of view, the loads of compressor motors are always the function of the rotor position and thus, they are repetitive. Several load curves for popular domestic compressors are shown in Fig. 1. We can find that the torque curve is nearly constant for scroll compressors but there is a significantly peak torque in the single-piston rotary compressor. In fact, such extremely alternating torque would contribute to the compressor speed ripple and thus, introduce the noise and vibrations to the overall system.

However, it is noted that high performance (i.e. low torque ripple) often implies high-production-cost. In order to promote variable-speed refrigeration systems to market as soon as possible, the low-cost single-piston rotary compressors become the only one solution. In order to less the effect of the positiondependent load on the overall system, the development of robust speed control becomes also very important. Many control methods had been developed to deal with the nonlinear load torque including the position-dependent loads [6-8]. However, the above methods are complex and not working in the sensorless operation.

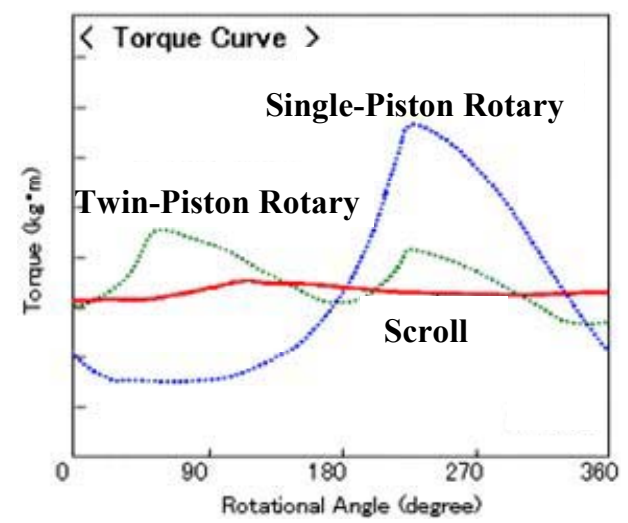

Fig. 1. Typical torque curve for various type of compressors. 
Consequently, we can find that the single-piston rotary compressors using BDCMs are preferred in the high-efficiency and low-cost variable-speed refrigeration systems. In our paper, a robust sensorless control has been developed and implemented to solve the following problems, such as sensorfree and position-dependent load.

The paper is organized as follows. In first, the effects of position-dependent load on the speed variations and the commutation intervals are studied. It shows that we can estimate the position-dependent load from the commutation intervals. Then, we introduce the used sensorless scheme based on sensing terminal voltage. Finally, simulated results have been given to demonstrate the robust performance of the proposed sensorless control for the position-dependent loads.

\section{Position-DePEndent Torque}

However, the actual rotating speed of the hermetic compressors in the refrigerant cycle system is not easy to be sensed. To demonstrate the proposed robust sensorless control, an experimental system, as shown in Fig. 2, is set up in our laboratory. It mainly consists of two identified BDCMs, an aluminum disk and a copper cylinder where the cylinder is fixed on the disk. All the motors and the disk are coupled to the same shaft. The former two BDCMs compose the known Motor-Generator (M-G) set where the terminals of the generator-end BDCM are connected to the Y-connected resistors in order to provide a constant load torque independent the rotor position. Besides, the copper cylinder on the disk will contribute to a position-dependent load because of the fixed down direction of the gravity force.

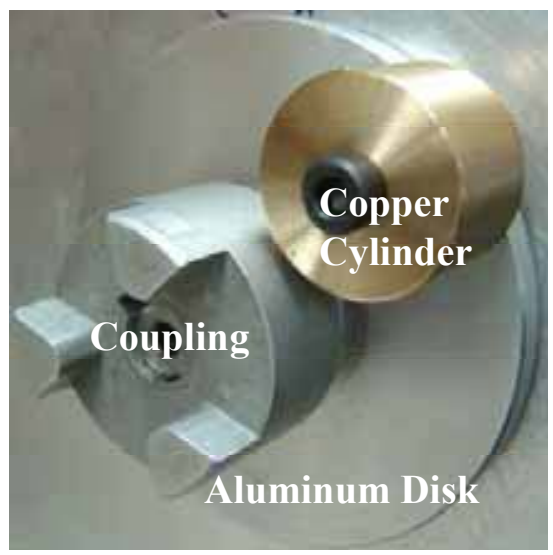

Fig. 2. Experimental position-dependent load.

To model the position-dependent characteristics in the experimental system, the illustrated plot for the three-phase Yconnected 4-pole BDCM is shown in Fig. 3 where only equivalent windings $a_{1}, a_{1}^{\prime}, a_{2}, a_{2}^{\prime}$ of phase $a$ are plotted and the mass of the copper cylinder is $M$. The stator winding $a_{1}$ is located at the fixed position $\theta_{r 0}$. The distance between the center of the aluminum disk and the mounting position of the cylinder is $R$. When the motor is rotating, all parts coupled to rotor are also rotating. Therefore, the gravity force $M g$ due to copper cylinder may either accelerate or decelerate the motor speed according to when the rotating rotor at the position $\theta_{r}$. It implies that the copper cylinder would contribute to a positiondependent load torque $T_{L, a c}\left(\theta_{r}\right)$

$$
T_{L, a c}\left(\theta_{r}\right)=M g R \cos \theta_{r}
$$

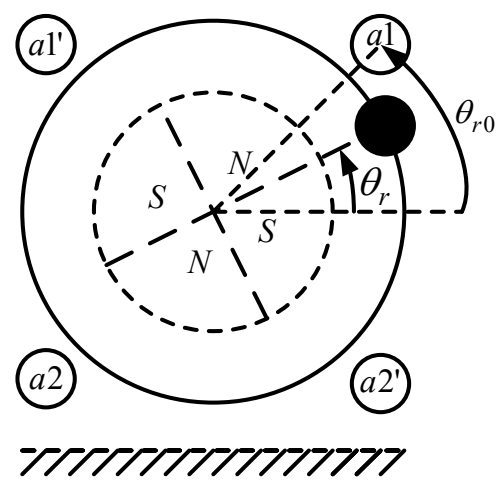

Fig. 3. Illustrated plot for position-dependent load.

From the mechanical equation, the generating torque $T_{e}$, the load torque $T_{L}$ and BDCM speed $\omega_{r}$ are related by

$$
T_{e}-T_{L}=J \frac{d \omega_{r}}{d t}+B \omega_{r}
$$

where $B$ is the damping coefficient ratio and $J$ is the total inertias of the rotor, the coupled disk and the copper cylinder. The load torque $T_{L}$ can be divided into dc component $T_{L, d c}$ and ac component $T_{L, a c}$ due to the position-dependent load torque.

For simplicity, the 4-pole BDCM is assumed to rotate at averaged speed $\omega_{r 0}$ and generate torque $T_{e}$ which can also be divided into dc component $T_{e, d c}$ and ac component $T_{e, a c}$. Then, in the steady state, the term $B \omega_{r 0}$, the dc torque components $T_{e, d c}$ and $T_{L, d c}$ in (2) can be cancelled. Therefore, (2) can be simplified and rewritten as

$$
T_{e, a c}\left(\theta_{r}\right)-T_{L, a c}\left(\theta_{r}\right)=-J \frac{d^{2} \theta_{r}}{d t^{2}}
$$

If the rotor position $\theta_{r}$ can be estimated, we can let the controllable motor torque component $T_{e, a c}$ vary with the function $\cos \left(\theta_{r}\right)$. Since the load torque component $T_{L, a c}$ is also the function of $\cos \left(\theta_{r}\right)$ from (1), the expression in (3) can be simplified as

$$
\Delta T \cos \left(\theta_{r}\right)=-J \frac{d^{2} \theta_{r}}{d t^{2}}
$$


However, from the final results, it is reasonable to assume that the rotor position can be estimated. From Fig. 3, we can express three electromotive forces $e_{a s}, e_{b s}$ and $e_{c s}$ as functions of rotor position $\theta_{r}$.

$$
\left\{\begin{array}{l}
e_{a s}=E \sin \left(2 \theta_{r}-2 \theta_{r 0}\right) \\
e_{b s}=E \sin \left(2 \theta_{r}+2 \pi / 3-2 \theta_{r 0}\right) \\
e_{c s}=E \sin \left(2 \theta_{r}+4 \pi / 3-2 \theta_{r 0}\right)
\end{array}\right.
$$

Some waveforms for the illustrated case $\left(\theta_{r 0}=-\pi / 12\right)$ are plotted in Fig. 4. It is noted that within each rotating cycle, there are twelve current commutations for 4-pole BDCM. However, by integrating (4), the rotor position $\theta_{r}(t)$ in $\mathrm{rads}$ and the varying motor speed $\omega_{r}$ in $\mathrm{rad} / \mathrm{sec}$ can be approximated as

$$
\begin{gathered}
\theta_{r}(t) \approx \omega_{r 0} t-\frac{\Delta T}{J \omega_{r 0}^{2}}\left(1-\cos \omega_{r 0} t\right) \\
\omega_{r}(t) \approx \omega_{r 0}-\frac{\Delta T}{J \omega_{r 0}} \sin \omega_{r 0} t
\end{gathered}
$$

In order to possess efficient torque capability, BDCMs must draw winding currents synchronizing with electromotive forces in (5). Therefore, for square-wave current, each commutation instant $t_{k}$ must occur at the rotor position equal to

$$
\theta_{r}\left(t_{k}\right)=2 \theta_{r 0}+\frac{k \pi}{6}, k \text { is integer }
$$

From (8), it is noted that the position difference between consequent commutation position $\theta_{r}\left(t_{k}\right)$ and $\theta_{r}\left(t_{k-1}\right)$ is constant and equal to $\pi / 6$. By using (6), this constant difference can be expressed as

$$
\frac{\pi}{6}=\omega_{r 0} T_{k}+\frac{\Delta T}{J \omega_{r 0}^{2}}\left[\cos \left(\omega_{r 0} t_{k}-\omega_{r 0} T_{k}\right)-\cos \left(\omega_{r 0} t_{k}\right)\right]
$$

where $T_{k}\left(=t_{k}-t_{k-1}\right)$ denotes the commutation interval between each commutation instant. By applying the common equality $\cos (A-B)=\cos A \cos B+\sin A \sin B$, (9) can be rewritten as

$$
\frac{\pi}{6}=\omega_{r 0} T_{k}+\frac{\Delta T}{J \omega_{r 0}^{2}} A\left[\cos \left(\omega_{r 0} t_{k}-\phi\right)\right]
$$

where $A=\sqrt{\left[\cos \left(\omega_{r 0} T_{k}\right)-1\right]^{2}+\sin \left(\omega_{r 0} T_{k}\right)^{2}}$ and

$$
\phi=\tan ^{-1}\left[\frac{\sin \left(\omega_{r 0} T_{k}\right)}{\cos \left(\omega_{r 0} T_{k}\right)-1}\right]
$$

In fact, the term $\cos \left(\omega_{r 0} T_{k}\right)$ is very close to $\cos (\pi / 6)=0.5$. Thus $A \approx 0.52$ and $\phi \approx 7 \pi / 12$. For simplicity, we can assume that

$$
\cos \left(\omega_{r 0} t_{k-m}\right) \approx \cos \left(\omega_{r 0} t_{k}-\frac{m \pi}{6}\right), m \text { is integer }
$$

Therefore, (10) can be rearranged as

$$
T_{k} \approx \frac{\pi}{6 \omega_{r 0}}-\frac{0.52 \Delta T}{J \omega_{r 0}^{3}}\left[\cos \left(\omega_{r 0} t_{k-3}\right)\right]
$$

From (13), we can find that the current commutation interval $T_{k}$ is a time-delay function of $\cos \left(\omega_{r 0} t_{k}\right)$. It means that the current position-dependent torque $\Delta T \cos \left(\omega_{r 0} t_{k}\right)$ can be observed from the future commutation period $T_{k+3}$. However, estimation from future information is not practical.

In steady state, position-dependent torque is repetitive, and each commutation interval can be assumed to be equal to the previous twelfth commutation interval $T_{k}=T_{k-12 p}$ where $p$ is an integer. Therefore, in order to obtain workable implementation, (13) can be simplified to obtain the practical equation

$$
\Delta T\left(\omega_{r 0} t_{k}\right) \propto \frac{\pi}{6}-\omega_{r 0} T_{k-9}
$$

where the current load torque $\Delta T \cos \left(\omega_{r 0} t_{k}\right)$ is proportional to the deviation of the product $\omega_{r 0} T_{k-9}$ from $\pi / 6$.

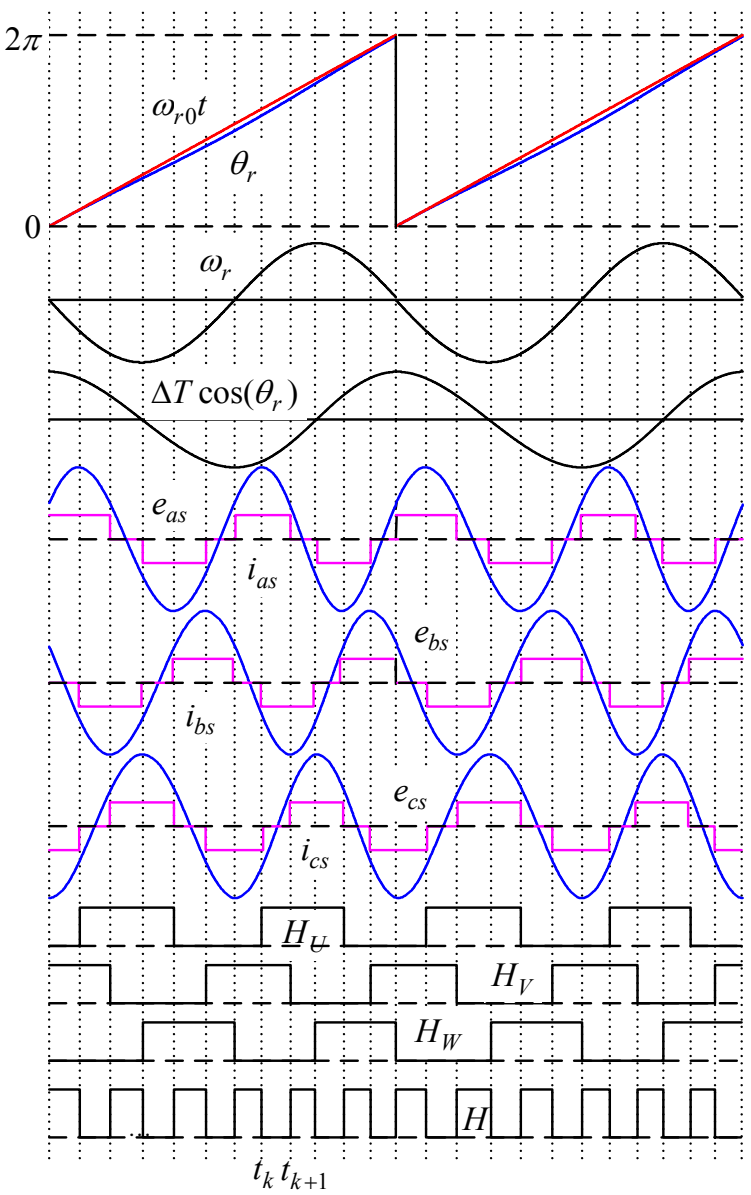

Fig. 4. Illustrated waveforms for 4-P BDCMs. 


\section{RoBUST SENSORLESS CONTROL}

The system configuration of the proposed robust sensorless control is plotted in Fig. 5 where the starting and control strategy and algorithms are implemented digitally in a 16-bits MCU SPMC75F2413A specifically designed for the variablespeed refrigeration system.

The used sensorless starting strategy can be divided into three modes: agitation mode, alignment mode and synchronization mode. Before the motor rotating in agitation mode, the sleeping refrigerant is initially agitated to be flowing easily, and then, the rotor is forced to locate in a given position in alignment mode with commutation state $\mathrm{F}$ shown in Table I. In synchronizing mode, the six commutation signals $G_{U} \sim G_{\bar{W}}$ start generating and changing with the series $\mathrm{A}=>\mathrm{B}=>\mathrm{C}=>$ $\mathrm{D}=>\mathrm{E}=>\mathrm{F} \Rightarrow \mathrm{A}$... to draw winding currents to compose a synchronous rotating magnetic field with increasing speed. Once the rotor reaches to some speed at which the sensorless circuits are able to function well, the speed loop turn to work to generate the six commutation signals according to the sensorless commutation table in Table I.

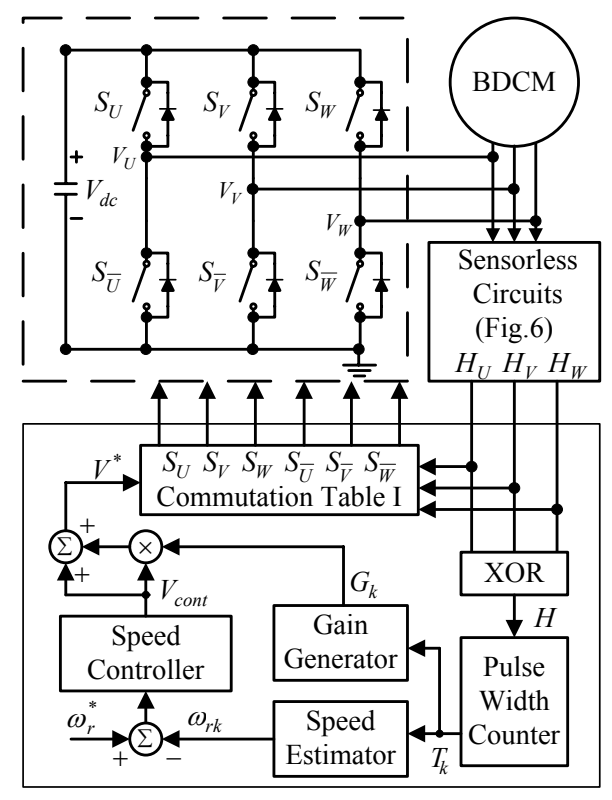

Fig. 5. System configuration of the proposed robust sensorless controller.

The alternative rotor position signals $H_{U}, H_{V}$ and $H_{W}$ are generated from sensing the terminal voltages $V_{U}, V_{V}$ and $V_{W}$ through the sensorless circuit as shown in Fig. 6. In order to commutate currents without any position sensor, the used sensorless circuit can be divided into three stages: $90^{\circ}$ phase shifting stage, dc filtering stage and PWM filtering stage. The first stage obtains alternative position signals from $90^{\circ}$ shifting the sensed floating terminal voltage by the low-pass filter. The latter two stages are designed to attenuate the undesired low-frequency and PWM components from the sensed terminal voltages [4].
Unlike the conventional speed loop where the controller output directly control the output voltage, an additional gain $G_{k}$ is included to vary the generating motor torque by tuning the output voltage $V^{*}$. After using the exclusive-or (XOR) operator, the three position signals are combined to single position signal $H$. Then, each commutation period $T_{k}$ can be directly obtained from counting the duration between each rising/falling edge of position signal $H$. For 4-pole BDCMs, the average speed $\omega_{r k}$ in rpm can be calculated from the commutation period $T_{k}$ by

$$
\omega_{r k}=\frac{5}{2 T_{k}}
$$

Based on (14), the profile of the position-dependent load torque $\Delta T\left(\theta_{r}\right)$ can be estimated from the commutation period $T_{k}$. It implies that by generating adequate gain $G_{k}$, we can vary the PWM duty $V^{*}$ and thus, change the winding currents and motor torque in order to oppose to the position-dependent load torque. For simplicity, the additional gain $G_{k}$ can be obtained from the simple equation

$$
G_{k}=W\left(\frac{T_{k-9}}{\sum_{n=0}^{11} T_{k-n}}-\frac{1}{12}\right)
$$

where the weighting factor $W$ is used to adjust the gain sensitivity to $T_{k}$. Then the PWM ratio $V^{*}$ is calculated as

$$
V^{*}=V_{\text {cont }}\left(1+G_{k}\right)
$$

Table I: Sensorless commutation table

\begin{tabular}{|c|c|c|c|c|c|c|c|}
\hline$\left(\begin{array}{ccc|c}H_{U} & H_{V} & H_{W}\end{array}\right)$ & $S_{U}$ & $S_{\bar{W}}$ & $S_{V}$ & $S_{\bar{U}}$ & $S_{W}$ & $S_{\bar{V}}$ & States \\
\hline$\left(\begin{array}{lll}0 & 1 & 1\end{array}\right)$ & OFF & ON & PWM & OFF & OFF & OFF & A \\
\hline$\left(\begin{array}{lll}0 & 1 & 0\end{array}\right)$ & PWM & ON & OFF & OFF & OFF & OFF & B \\
\hline$\left(\begin{array}{lll}1 & 1 & 0\end{array}\right)$ & PWM & OFF & OFF & OFF & OFF & ON & C \\
\hline$\left(\begin{array}{lll}1 & 0 & 0\end{array}\right)$ & OFF & OFF & OFF & OFF & PWM & ON & D \\
\hline$\left(\begin{array}{lll}1 & 0 & 1\end{array}\right)$ & OFF & OFF & OFF & ON & PWM & OFF & E \\
\hline$\left(\begin{array}{lll}0 & 0 & 1\end{array}\right)$ & OFF & OFF & PWM & ON & OFF & OFF & F \\
\hline
\end{tabular}

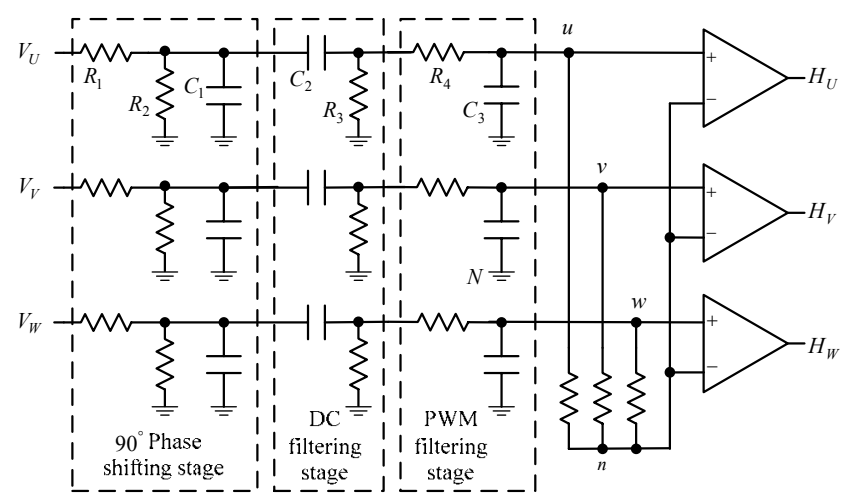

Fig. 6. Sensorless circuits 


\section{Simulated Results}

From (17) and Fig. 5, we can find that the proposed sensorless control with zero duty gain $G_{k}=0$ is analogous to the traditional speed control where the PWM ratio is equal to the speed controller output $V^{*}=V_{\text {cont }}$. The simulated waveforms for $G_{k}=0$ are plotted in Fig. 7 where the proportional-integral (PI) type is used in the speed controller. Some nominal values and circuit elements are listed in Table I.

\begin{tabular}{|c|c|}
\hline Stator resistance & $0.7 \Omega$ \\
\hline Stator inductance & $\begin{array}{l}L q=10.5 m H \\
L d=4 m H\end{array}$ \\
\hline $\begin{array}{l}\text { Voltage constant } \\
\text { (Line-to-Line) }\end{array}$ & $23.63 \mathrm{~m} V_{r m s} / \mathrm{rpm}$ \\
\hline Pole number & 4 pole \\
\hline Motor inertia & $0.0004 \mathrm{~kg}-\mathrm{m}^{2}$ \\
\hline DC link voltage & $300 \mathrm{~V}$ \\
\hline PWM frequency & $5 \mathrm{kHz}$ \\
\hline DC load torque & $1 N-m$ \\
\hline AC load torque & $0.5 N-m$ (peak) \\
\hline
\end{tabular}

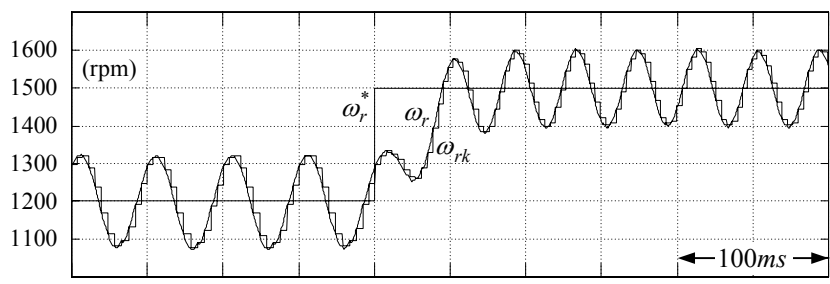

(a)

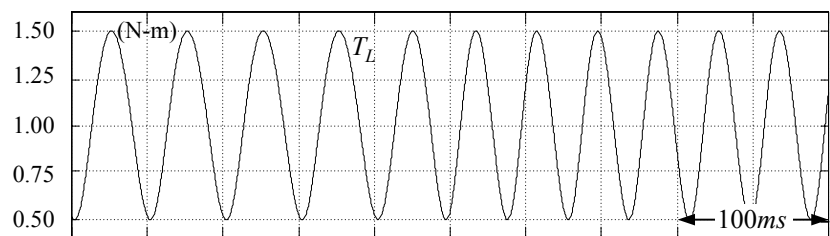

(b)

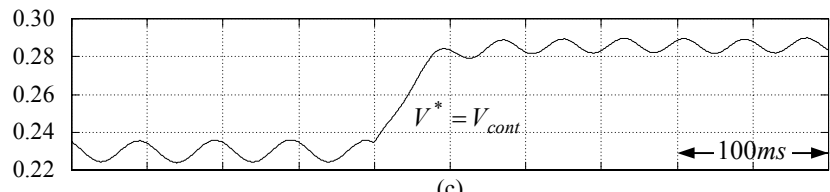

(c)

Fig. 7. Simulated waveforms with zero gain $G_{k}=0$ due to speed command $\omega_{r}^{*}$ changing from 1200rpm to 1500rpm: (a) actual speed $\omega_{r}$ and the estimated speed $\omega_{r k}$, (b) load torque $T_{L}$ and (c) PWM ratio $V^{*}$.

From Fig. 7(a), we can find that the actual speed $\omega_{r}$ deviates from the speed command $\omega_{r}^{*}$ due to the repetitive positiondependent load torque in Fig. 7(b). Owing to the speed estimation delay at high speed is smaller than that at low speed, the speed ripples are about $120 \mathrm{rpm}$ and $100 \mathrm{rpm}$ at $\omega_{r}^{*}=1200 \mathrm{rpm}$ and $\omega_{r}^{*}=1500 \mathrm{rpm}$, respectively. In Fig. 7(c), it is noted that the PWM duty $V^{*}$ fluctuates according to the speed error between the speed command and the speed estimation. In addition, the PWM duty $V^{*}$ is almost at its valley and peak when the load torque is near its maximum and minimum values, respectively. However, such adversity would contribute to aggravate the speed ripple. Therefore, we propose the robust sensorless control to alleviate the speed ripple due to the position-dependent load torque.

To evaluate the proposed robust sensorless control, some simulated results with the same controller parameter as Fig. 7 are illustrated in Fig. 8 where nonzero gain $G_{k}$ is included and the final PWM duty is calculated from (17). Compared with the speed in Fig. 7(a), the speed variation in Fig. 8(a) is reduced to about $80 \mathrm{rpm}$ based on the same speed estimation delay. It implies that the proposed algorithms in (15) through (17) make the sensorless operation possess the robust speed performance to the position-dependent load torque.

From Fig. 8(b), the duty gain $G_{k}$ provides useful estimation of the load torque $T_{L}$, which can help to reverse the adversity in Fig. 7(b) and (c). That is, complying with the system stability, we can adequately set the weighing factor $W$ in (16) as large as possible to adjust the final PWM duty $V^{*}$ in phase with the load torque variations. From Fig. 8(c), we can find that the final PWM duty $V^{*}$ fluctuates effectively in order to vary the generating torque at the right moments.
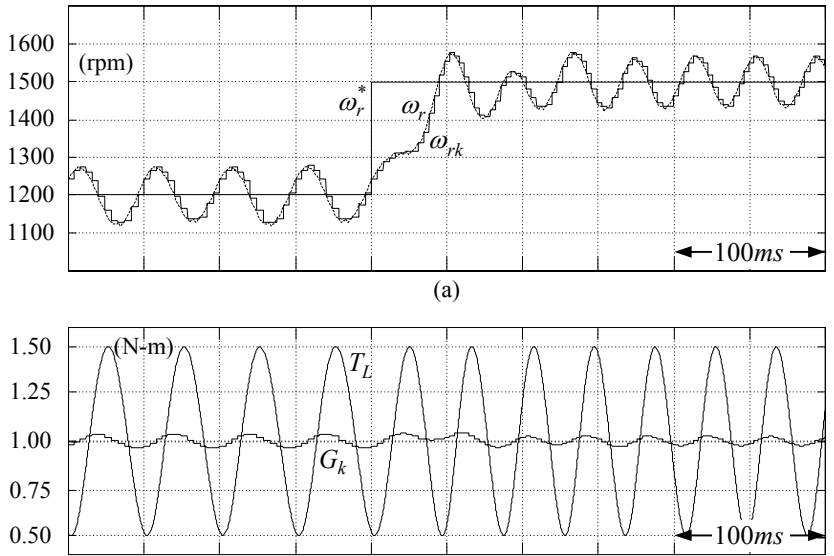

(b)

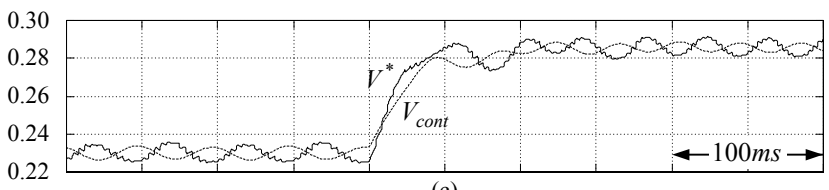

(c)

Fig. 8. Simulated waveforms due to speed command $\omega_{r}^{*}$ changing from $1200 \mathrm{rpm}$ to $1500 \mathrm{rpm}$ : (a) actual speed $\omega_{r}$ and the estimated speed $\omega_{r k}$, (b) load torque $T_{L}$ and duty gain $G_{k}$ and (c) PWM ratio $V^{*}$ and speed controller output $V_{\text {cont }}$.

\section{CONCLUSIONS}

Without any position sensors, the motor terminal voltages were sensed to generate the helpful position signals to correctly commutate winding currents. A mathematical model of repetitive position-dependent load torque was developed in this paper. The model was then used to obtain the algorithms of the proposed robust sensorless control where the PWM duty was 
not only dependent on the speed controller output but also on the yielded duty gain. These algorithms were very simple and practical to be implemented in DSP/MCU based system. Simulated results had demonstrated the proposed robust sensorless control. The developed control can be advanced by using other algorithms to further condense the speed ripple.

\section{REFERENCES}

[1] H. Murakami, Y. Honda, H. Kiriyama, S. Morimoto, and Y. Takeda, "The performance comparison of SPMSM, IPMSM and SynRM in use as air-conditioning compressor," in Proc. IAS'99, pp. 840-845, 1999.

[2] S. Ogasawara, and H. Akagi, "An approach to position sensorless drive for brushless DC motors," IEEE Trans. Ind. Applicat., vol.27, no. 5, pp. 928-933, 1991.

[3] H. C. Chen, and C. M. Liaw, "Current-mode control for sensorless BDCM drive with intelligent commutation tuning," IEEE Trans. Power Electron., vol. 17, no. 5, pp. 747-756, 2002.

[4] H. C. Chen, Y. C. Chang, and C. K. Huang, 'Practical Sensorless Control for Inverter-Fed BDCM Compressors,' IET Proc. Electric Power Applications, vol. 1, no. 1, pp. 127-132, Jan. 2007.

[5] E. Delaleau and A. M. Stankovic, "Modeling and Simulation of the Induction Motor with Position-Dependent Load Torque," in Proceedings of the 42 $\mathrm{P}^{n d \mathrm{P}}$ Conference on Decision and Control, pp. 6212-6217, 2003.

[6] J. Solsona and M. I. Valla, and C. Muravchik, "Nonlinear Control of a Permenent Magnet Synchronous Motor with Disturbance Torque Estimation," IEEE Trans. Energy Conversion, vol. 15, no. 2, pp. 163-168, Jun. 2000,

[7] Z. Kovacic, S. Bogdan and M. Balenovic, "A Model Reference \& Modelbased Self-learning Fuzzy Logic Controller as a solution for Control of Nonlinear Servo System," IEEE Trans. Energy Conversion, vol. 14, no. 5, pp. 1479-1484, Dec. 1999.

[8] P. Krishnamurthy, W. Lu, and A. Keyhani, "A Robust Force Controller for an SRM Based Electromechanical Brake System," in Proceedings of the 44th Conference on Decision and Control, pp. 2006-2011, 2005. 